\title{
Establish a Nomogram of Cardiac Postoperative Cognitive Dysfunction
}

\author{
Naxin Xie, MD, Sunang Yan, MD, Xuejun Sun, MD, Haibo Liu, MD \\ Department of Cardiovascular Surgery, Fujian Medical University Affiliated First Quanzhou Hospital, Quanzhou, China
}

\section{ABSTRACT}

Objectives: Cardiac postoperative cognitive dysfunction (c-POCD) is a common complication. This article established a nomogram by analyzing preoperative and intraoperative data to help identify high-risk patients and take effective management for prevention of c-POCD in early stage.

Measurements and main results: A total of 265 patients were enrolled in this study, 27 of whom were diagnosed as cardiac postoperative cognitive dysfunction (c-POCD). Patients were divided into a control group and c-POCD group. Univariate analysis suggested that gender, smoking, drinking history, hypertension, white blood cell (WBC) count, aspartate aminotransferase (AST), high-sensitivity troponin (hs-CRP), arrhythmia, left atrial diameter (LAD), cardiopulmonary bypass $(\mathrm{CPB})$ time, and the ascending aortic block (AAB) time were correlated with postoperative cognitive dysfunction after cardiac surgery. Multivariate regression analysis indicated that $\mathrm{CPB}$ time $(P=0.0015$, OR $(95 \% \mathrm{CI})=6.696(2.068-21.675)$, hypertension $(P=0.0098$, OR $(95 \% \mathrm{CI})=3.776(1.377-10.356)$, WBC count $(P=0.0227$, OR $(95 \% \mathrm{CI})=3.358(1.184-9.522)$, AST $(P=0.0128$, OR $(95 \% \mathrm{CI})=3.966(1.340-11.735)$, and arrhythmia $(P=0.0017$, OR $(95 \% \mathrm{CI})=5.164(1.855-14.371)$ were the independent risk factors of cognitive dysfunction after cardiac surgery and used to establish a nomogram for clinical use. The initial C-index of the nomogram was 0.8182 and good calibration. Corrected C-index value of 0.793 was reached after internal validation. The area under ROC curve of this model was 0.8188 (95\%CI: $0.7185-0.9190)$. The positive odds ratio (PLR) was 1.21 (95\%CI: 1.1-1.3), and the negative odds ratio (NLR) was 0.18 (95\% CI: 0.03-1.3).

Conclusion: This nomogram incorporating the $\mathrm{CPB}$ time, hypertension, WBC count, AST, and arrhythmia to predict the risk of c-POCD. The internal validation shows a good forecasting effect.

\section{INTRODUCTION}

Cardiac postoperative cognitive dysfunction (c-POCD) is receiving increasing attention, with an incidence of

Received December 15, 2020; received in revised form fanuary 30, 2021; accepted February 1, 2021

Correspondence: Haibo Liu, MD, Department of Cardiovascular Surgery, Fujian Medical University Affiliated First Quanzhou Hospital, 362000 Quanzhou, China (e-mail: lbb2233.hot@163.com)
13.5\%-41.7\% [Koster 2008; Mangusan 2015; Zhang 2015]. It always shows symptoms of alienation, anxiety, delirium, memory impairment, personality change, and changes in social integration capabilities. As a result, patients often do not cooperate in diagnosis and treatment, and the treatment is difficult to conduct. However, the etiology and pathogenesis of c-POCD are still unclear, and it may include surgery-, anesthesia- and patient-related factors [van Harten 2012]. Once cognitive dysfunction after cardiac surgery occurs, it is difficult to control and reverse, and the treatment effect often is unsatisfactory. Therefore, it is important to improve the identification and quantification of risk factors for c-POCD, as this will assist clinicians and patients in the decision-making process, when invasive procedures are planned.

Nomogram was introduced to oncology research by Kattan from Memorial Sloan-Kettering Cancer Center in 1998 to predict the recurrence rate after radical prostatectomy. Due to its simple and intuitive characteristics, nomogram now widely has been used in the prognostic analysis of various diseases [Iasonos 2008; Kawai 2015].

This article was a retrospective research to screen the risk factors for c-POCD and establish a nomogram to help identify high-risk patients early and then provide timely intervention.

\section{PATIENTS AND MEASURMENTS}

A total of 265 patients who underwent routine open cardiac surgery [Yuan 2012; Di Tomasso 2015; Hessel 2015] in Fujian Medical University Affiliated First Quanzhou Hospital from January 2015 to January 2018 were enrolled in this study. Twenty-seven of these patients were diagnosed with cardiac postoperative cognitive dysfunction (c-POCD), and they were divided into a control group and an experimental group. Patients who underwent valve surgery and coronary artery bypass grafting (CABG) were included our research. Exclusion criteria: (1) non-routine open cardiac surgery; (2) clinical data missing; (3) perioperative cognitive dysfunction; (4) psychotropic drugs abuse and alcoholism; (5) suffer from mental illness or family history of mental illness. Data such as demographic, diseases, preoperative examinations, CPB time, and the ascending aortic block (AAB) time of the included patients were collected from medical records. (Table 1)

Assessment of cardiac postoperative cognitive function: This study refers to the Montreal Cognitive Assessment (MoCA) [Nasreddine 2005] and Mini-mental state (MMSE) [Folstein 1975] assessment scale to evaluate cardiac postoperative cognitive function from orientation, attention, memory, execution, language and visual-spatial functions. c-POCD 
Table 1. Baseline demographics of included patients

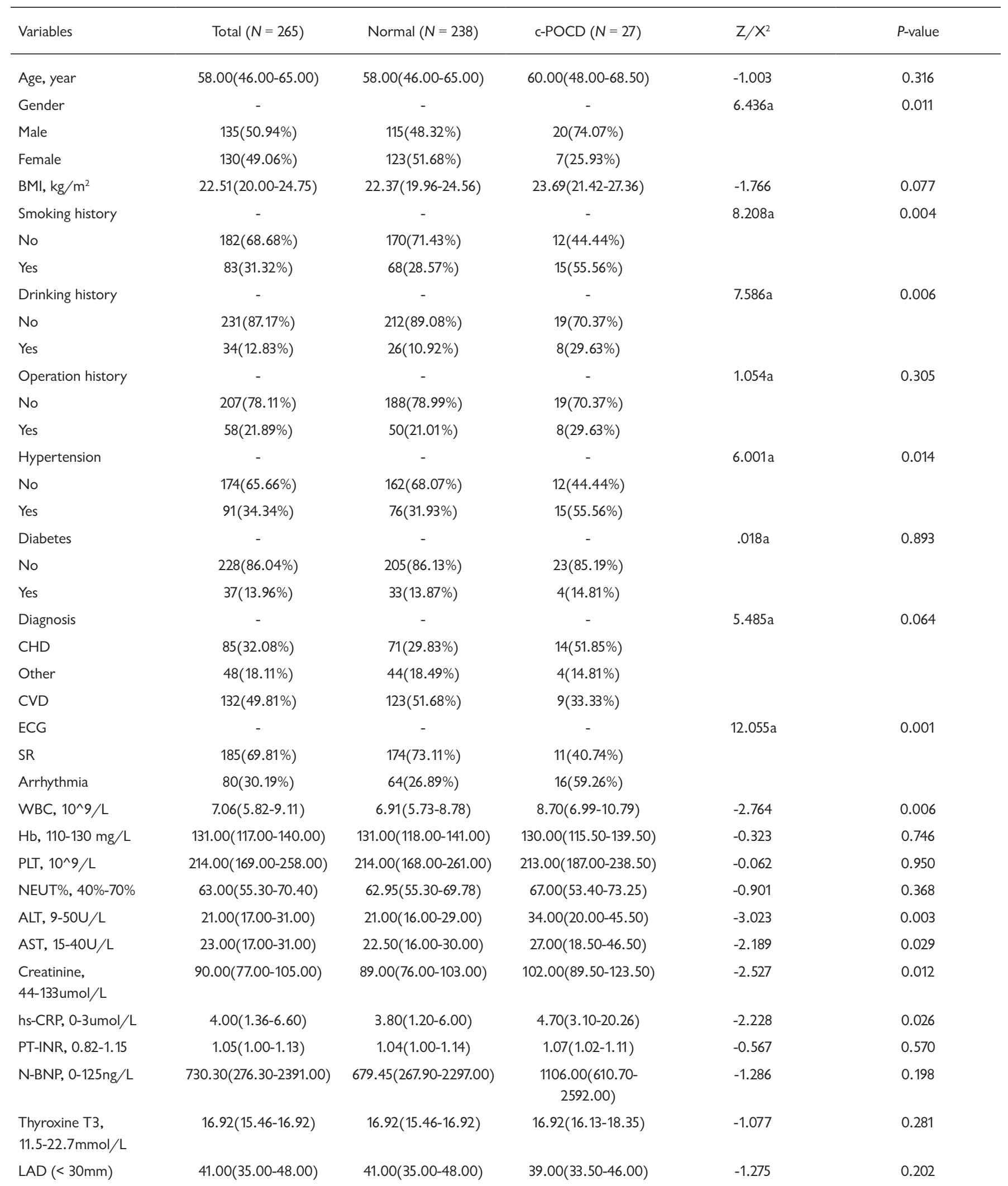




\begin{tabular}{lcccccc} 
LVD $(45-55 \mathrm{~mm})$ & $52.00(46.00-58.00)$ & $52.00(46.00-58.00)$ & $54.00(48.00-58.00)$ & -1.154 & 0.249 \\
RVD $(7-23 \mathrm{~mm})$ & $21.00(19.00-23.00)$ & $20.00(19.00-23.00)$ & $21.00(20.00-23.00)$ & -1.493 & 0.135 \\
LVEF $(\geq 55 \%)$ & $63.00(54.00-68.00)$ & $63.00(54.00-68.00)$ & $59.00(52.50-65.50)$ & -1.114 & 0.265 \\
CPB time(min) & $127.70(96.00-155.00)$ & $123.00(95.00-148.00)$ & $169.00(136.00-199.50)$ & -4.474 & 0.000 \\
AAB time $(\min )$ & $84.00(63.00-108.00)$ & $81.00(61.00-103.00)$ & $110.00(87.00-132.00)$ & -3.931 & 0.000 \\
\hline
\end{tabular}

c-POCD, cardiac postoperative cognitive dysfunction; BMI, body mass index; CHD, coronary atherosclerotic heart disease; OTHER, CHD + CVD; CVD, valvular heart disease; ECG, electrocardiogram; SR, sinus rhythm; WBC, white blood cell count; Hb, Hemoglobin; PLT, platelet count; NEUT\%, neutrophil percentage; ALT, alanine aminotransferase; AST, aspartate aminotransferase; hs-CRP, high-sensitivity C-reactive protein; PT-INR, International standard ratio of prothrombin time; N-BNP, N-terminal brain natriuretic peptide; LAD, left atrial diameter; LVD, left ventricular diameter; RVD, right ventricular diameter; LVEF, left ventricular ejection fraction; $\mathrm{AAB}$, ascending aortic block

was diagnosed when any one of the symptoms occurs: (1) delirium; (2) language disorder; (3) persecuted delusion; (4) indifference; (5) memory disorder.

Statistical analysis: All statistical analyses were performed with SPSS (version 23.0) and R (Version 3.5.3; The R Foundation for Statistical Computing, Vienna, Austria) software. The measurement data were expressed as mean \pm standard deviation $\left(X_{ \pm} S\right)$, group $t$ test was used for comparison among groups, and chi-square test was used for comparison of count data. Risk factor analysis: First, single factor analysis was used to screen out possible risk factors, then logistic multivariate regression analysis was used to screen out independent risk factors, and the "rms" package of R language was used to establish the nomogram. Bootstrap $(b=1000)$ was used for internal validation of the nomogram, and the corrected C-index was used to express the effect of the nomogram. All statistical tests were bilateral tests and were of statistical significance $(P<0.05)$.

\section{RESULTS}

The demographics of included patients: The postoperative cognitive function of 265 patients in this research was analyzed, and 27 patients were diagnosed c-POCD. Univariate analysis results showed that the patient's gender, smoking history, drinking history, hypertension, white blood cell count (WBC), aspartate aminotransferase (AST), high-sensitivity troponin (hsCRP), arrhythmia, left atrial diameter (LAD), cardiopulmonary bypass $(\mathrm{CPB})$ time, and the ascending aortic block (AAB) time were significantly correlated with $c-P O C D(P<0.05)$, and other indicators had no significant difference. (Table 2)

Multivariate regression analysis screen independent risk factors for c-POCD: The results of univariate analysis were incorporated into the multivariate regression analysis, which showed that $\mathrm{CPB}$ time $(P=0.0015, \mathrm{OR}(95 \% \mathrm{CI})=6.696(2.068-21.675)$, hypertension $(P=0.0098, \mathrm{OR}(95 \% \mathrm{CI})=3.776(1.377-10.356)$, WBC $(P=0.0227$, OR $(95 \% \mathrm{CI})=3.358(1.184-9.522)$, AST $(P$ $=0.0128$, OR $(95 \% \mathrm{CI})=3.966(1.340-11.735)$, and arrhythmia $(P=0.0017, \mathrm{OR}(95 \% \mathrm{CI})=5.164(1.855-14.371)$ were independent risk factors for c-POCD $(P<0.05)$. (Table 3$)$

Establish a nomogram to predict the occurrence of c-POCD: Based on the results of multivariate regression analysis, a nomogram was established to predict the probability

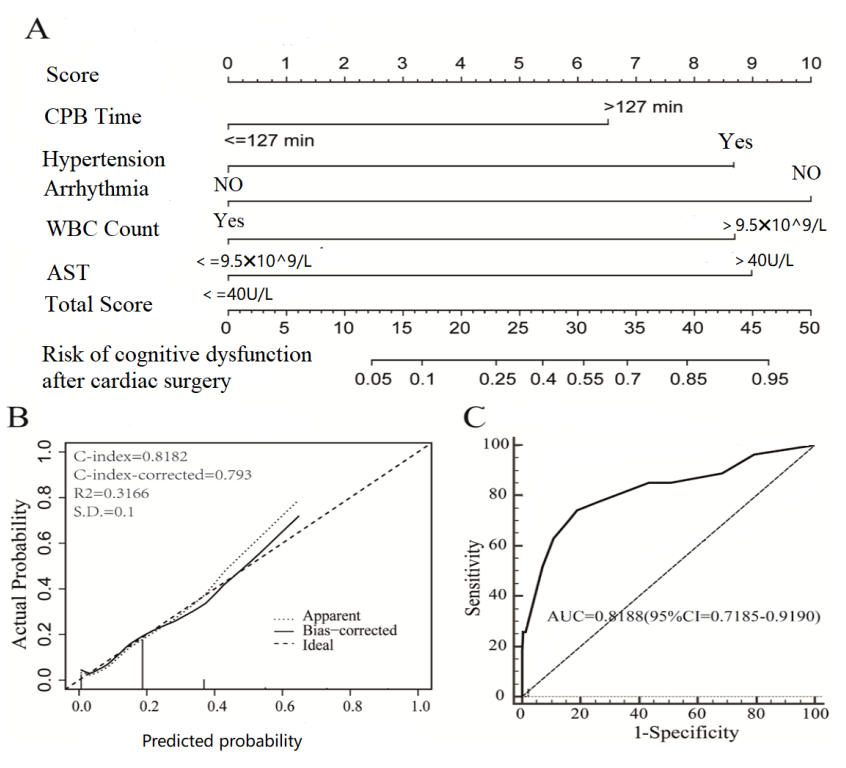

Figure. A) A nomogram of cardiovascular postoperative cognitive dysfunction; B) The intra-group verification result of the nomogram; C) The ROC curve of nomogram

c-POCD. (Figure A) The initial C-index of the nomogram was 0.8182 and good calibration. Corrected C-index value of 0.793 was reached after bootstrap internal validation $(b=1000)$. (Figure B) The area under ROC curve of this model was 0.8188 (95\%CI: 0.7185-0.9190). (Figure C) This suggested that the nomogram has a good predictive value for c-POCD. The positive odds ratio (PLR) was 1.21 (95\% CI: 1.1-1.3), and the negative odds ratio (NLR) was 0.18 (95\%CI: 0.03-1.3).

\section{DISCUSSION}

In 1955, Bedford proposed the concept of postoperative cognitive dysfunction. Postoperative cognitive dysfunction is also a common complication of cardiac surgery, which often results in patients not cooperating in treatment, the increase of postoperative mortality, extension of hospital stays, and waste of more medical resources. Therefore, early intervention is very important for high-risk patients [Gottesman 2010; 
Table 2. Univariate analysis between baseline characteristics and c-POCD

\begin{tabular}{|c|c|c|c|c|c|c|}
\hline Age $(>58 y)$ & $117(49.16 \%)$ & $15(55.56 \%)$ & 0.257 & 0.408 & $1.293(0.581-2.878)$ & 0.5296 \\
\hline $\mathrm{BMI}(>22.5)$ & $116(48.74 \%)$ & $16(59.26 \%)$ & 0.425 & 0.413 & $1.530(0.681-3.434)$ & 0.3028 \\
\hline Smoking (yes) & $68(28.57 \%)$ & $15(55.56 \%)$ & 1.139 & 0.413 & $3.125(1.391-7.021)$ & 0.0058 \\
\hline Operation history (yes) & $50(21.01 \%)$ & $8(29.63 \%)$ & 0.459 & 0.451 & $1.583(0.655-3.828)$ & 0.3078 \\
\hline Hypertension (yes) & $76(31.93 \%)$ & $15(55.56 \%)$ & 0.980 & 0.411 & $2.664(1.189-5.969)$ & 0.0172 \\
\hline Diabetes (yes) & $33(13.87 \%)$ & $4(14.81 \%)$ & 0.077 & 0.573 & $1.080(0.351-3.323)$ & 0.8927 \\
\hline Diagnosis & - & - & - & - & - & 0.0742 \\
\hline $\mathrm{CHD}$ & $71(29.83 \%)$ & $14(51.85 \%)$ & - & - & 1 & \\
\hline PLT, >125×10^9/L & $14(5.88 \%)$ & $3(11.11 \%)$ & 0.693 & 0.671 & $2.000(0.536-7.458)$ & 0.3020 \\
\hline NEUT\%\%, >70\% & $70(29.41 \%)$ & $11(40.74 \%)$ & 0.501 & 0.417 & $1.650(0.729-3.734)$ & 0.2295 \\
\hline $\mathrm{ALT},>50 \mathrm{U} / \mathrm{L}$ & $18(7.56 \%)$ & $5(18.52 \%)$ & 1.022 & 0.553 & $2.778(0.940-8.208)$ & 0.0646 \\
\hline AST, $>40 U / L$ & $24(10.08 \%)$ & $10(37.04 \%)$ & 1.657 & 0.453 & $5.245(2.159-12.744)$ & 0.0003 \\
\hline Creatinine, $>133 \mathrm{umol} / \mathrm{L}$ & $23(9.66 \%)$ & $5(18.52 \%)$ & 0.754 & 0.542 & $2.125(0.735-6.144)$ & 0.1643 \\
\hline hs-CRP, >3umol// & $141(59.24 \%)$ & $20(74.07 \%)$ & 0.890 & 0.410 & $2.434(1.090-5.437)$ & 0.0300 \\
\hline PT-INR, >1.15 & $55(23.11 \%)$ & $4(14.81 \%)$ & -0.547 & 0.563 & $0.579(0.192-1.745)$ & 0.3313 \\
\hline N-BNP, >125ng/L & $211(88.66 \%)$ & $24(88.89 \%)$ & 0.023 & 0.646 & $1.024(0.289-3.628)$ & 0.9711 \\
\hline CPB time $(>127 \mathrm{~min})$ & $23(9.66 \%)$ & $16(59.26 \%)$ & 2.211 & 0.558 & $9.125(3.058-27.232)$ & 0.0001 \\
\hline $\mathrm{AAB}$ time $(>84 \mathrm{~min})$ & $20(8.40 \%)$ & $12(44.44 \%)$ & 1.252 & 0.458 & $3.498(1.425-8.585)$ & 0.0063 \\
\hline
\end{tabular}

c-POCD, cardiac postoperative cognitive dysfunction; BMI, body mass index; CHD, coronary atherosclerotic heart disease; Other, CHD + CVD; CVD, valvular heart disease; WBC, white blood cell count; Hb, Hemoglobin; PLT, platelet count; NEUT\%, neutrophil percentage; ALT, alanine aminotransferase; AST, aspartate aminotransferase; hs-CRP, high-sensitivity C-reactive protein; PT-INR, International standard ratio of prothrombin time; N-BNP, N-terminal brain natriuretic peptide; LAD, left atrial diameter; LVD, left ventricular diameter; RVD, right ventricular diameter; LVEF, left ventricular ejection fraction; CPB, cardiopulmonary bypass; $A A B$, ascending aortic block

Koster 2012]. However, there is no prediction model to estimate the risk of c-POCD. This research established a nomogram, which has good predictive value. Compared with traditional prediction models, the nomogram is easier to be used by clinicians and also can achieve individualized predictions.

This research reported an occurrence probability of c-POCD of $10.18 \%$, which is close to the probability reported in past research [Koster 2008; Mangusan 2015; Zhang 2015].
The etiology and pathogenesis of c-POCD are unclear so far and are closely related to preoperative, intraoperative, and surgical factors. General diagnosis and treatment methods often lead to delay in treatment. At present, it is believed that c-POCD is induced by surgery and anesthesia and is a kind of neurological hypofunction caused by the combined action of multiple factors, involving the disorders of central nervous system, endocrine, and immune systems [Bi 2014]. 
Table 3. Multivariate regression analysis screen independent risk factors of c-POCD

\begin{tabular}{lcccc}
\hline Variables & B & S.E. & OR $(95 \% \mathrm{Cl})$ & $P$-value \\
\hline CPB time, $>127$ min & 1.901 & 0.599 & $6.696(2.068-21.675)$ & 0.0015 \\
Hypertension & 1.329 & 0.515 & $3.776(1.377-10.356)$ & 0.0098 \\
AST, $>40 \mathrm{U} / \mathrm{L}$ & 1.378 & 0.554 & $3.966(1.340-11.735)$ & 0.0128 \\
Arrhythmia & 1.642 & 0.522 & $5.164(1.855-14.371)$ & 0.0017 \\
WBC, $>9.5 \times 10^{\wedge} 9 / \mathrm{L}$ & 1.211 & 0.532 & $3.358(1.184-9.522)$ & 0.0227 \\
\hline
\end{tabular}

c-POCD, cardiac postoperative cognitive dysfunction; CPB, cardiopulmonary bypass; AST, aspartate aminotransferase; WBC, white blood cell count

Univariate analysis found that age and diabetes history were of no statistical significance. However, many studies showed that age was a main risk factor for the occurrence of c-POCD [Canet 2003; Evered 2011; Gao 2005]. In addition, the research of Launer also showed that diabetes was associated with c-POCD [Launer 1993; Shaw 2003]. This may be due to the sample size not being large enough to detect weak statistical correlations.

Although gender, smoking history, drinking history, highsensitivity troponin (hs-CRP), arrhythmia, left atrial diameter (LAD), and ascending aortic block (AAB) time were related to the c-POCD in univariate analysis, they were not statistically significant in multivariate regression analysis, so they were not included in the prediction model. The nomogram showed that the patients with longer CPB time, hypertension, arrhythmia, higher WBC count, and AST were more likely to have c-POCD.

$\mathrm{CPB}$ is a non-physiological process. It is common practice for the arterial pressure to be maintained at around $60 \mathrm{mmHg}$, which may disrupt cerebral autoregulation, particularly in patients with a history of hypertension, eventually leading to poor cerebral perfusion and oxygenation and c-POCD. At present, it is not possible to directly measure intraoperative cerebral blood flow or perfusion. Real-time assessment of regional (frontal) cortical oxygenation by near-infrared spectroscopy is promising technology, which offers a non-invasive method of assessing cerebral haemoglobin oxygen saturation, thereby giving an indication of the overall balance between oxygen delivery, requirements, and uptake. It thus has the potential to demonstrate untoward intraoperative consequences of off-pump surgery. Early work also suggests that the technique can be used to assess cerebral autoregulation [Brady 2010]. On the other hand, on-pump cardiac surgery induces a widespread systemic inflammatory response that is associated with c-POCD [Hudetz 2011], which ultimately leads to cognitive dysfunction [Kalman 2006; Wan 2007]. In addition, the permeability of the blood-brain barrier will increase under pathological conditions; the inflammatory factors can through the blood-brain barrier to result in c-POCD [Rasmussen 2006; Wan 2010]. Therefore, immune system modulation is required to further work, not only an antiinflammatory one, such as the corticosteroid dexamethasone, but also by other agents that may exert beneficial effects on the balance between pro- and anti-inflammatory mediators, such as interleukin-6 or tumor necrosis factor-a, and interleukin-4 or interleukin-10, respectively.

In this article, arrhythmia is considered to be the strongest independent risk factor for cognitive dysfunction after cardiac surgery, particularly in atrial fibrillation and cognitive dysfunction after cardiac surgery. Its possible mechanisms mainly follow three aspects: First, abnormal fluctuating rhythm often leads to insufficient stroke volume, which leads to insufficient cerebral perfusion and c-POCD [Cacciatore 2012]; second, the microthrombi in the atrium fall during the preoperative and intraoperative operations and block the cerebral microvessels, causing c-POCD [Richards 1997]; and third, the use of anticoagulant drugs before the operation can cause tiny hemorrhagic foci in the brain. Unfortunately, there currently are no effective measures to prevent the c-POCD of patients with arrhythmia [Wu 2016; Akoudad 2014]. Although in our study, AST is an independent risk factor for c-POCD, there is little past research. This may be because abnormal liver function often leads to slow metabolism of anesthetic drugs and eventually c-POCD [Bucak 2014; Silverstein 2007].

In addition, this research also found that the patients with hypertension had higher occurrence of c-POCD. This may be related to the cerebral ischemia caused by the decrease in the mean arterial pressure of the systemic circulation during the operation. The patient's blood pressure is maintained at a high level for a long time, the perfusion pressure under CPB is low during operation, and patients with long-term hypertension have cerebral arteriosclerosis, poor vascular elasticity, and weakened autonomic regulation [Heyer 2002; Monk 2008], which will increase the risk of c-POCD.

There currently are no safe and effective interventions for c-POCD. Adequate preoperative education can significantly reduce the probability of cognitive dysfunction after surgery [Gao 2005]. In addition, correcting the inflammation and abnormal liver function before the operation also is of great significance for preventing cognitive dysfunction after cardiac surgery [Bi 2014].

\section{LIMITATIONS}

There are several of limitations of this research. First, we only collected data from a single center, without a large sample. Second, this research is a retrospective analysis, and 
many data cannot be further verified. Third, anesthesiarelated factors are considered to be independent risk factors for c-POCD, but we have not been able to provide detailed anesthesia records. All in all, it requires multi-center, largesample, and prospective study in the future.

\section{CONCLUSION}

In our research, CPB time, hypertension, arrhythmia, WBC, and AST level were independent risk factors for c-POCD. Based on these five factors, the nomogram established has higher accuracy in predicting c-POCD. Compared with the traditional prediction models, the nomogram is more intuitive and convenient for the clinicians.

\section{ACKNOWLEDGEMENTS}

The authors are supported by grants from the Quanzhou Science and Technology Project: 2018C065R.

\section{REFERENCES}

Akoudad S, Darweesh SK, Leening MJ, et al. 2014. Use of coumarin anticoagulants and cerebral microbleeds in the general population. Stroke; a journal of cerebral circulation. 45(11):3436-3439.

Bi Y, Liu S, Yu X, Wu M, Wang Y. 2014. Adaptive and regulatory mechanisms in aged rats with postoperative cognitive dysfunction. Neural regeneration research. 9(5):534-539.

Brady K, Joshi B, Zweifel C, et al. 2010. Real-time continuous monitoring of cerebral blood flow autoregulation using near-infrared spectroscopy in patients undergoing cardiopulmonary bypass. Stroke. 41(9):1951-1956.

Bucak N, Begec Z, Erdil F, et al. 2014. Postoperative cognitive dysfunction in living liver transplant donors. Experimental and clinical transplantation: official journal of the Middle East Society for Organ Transplantation. 12 Suppl 1:81-85.

Cacciatore F, Testa G, Langellotto A, et al. 2012. Role of ventricular rate response on dementia in cognitively impaired elderly subjects with atrial fibrillation: a 10 -year study. Dementia and geriatric cognitive disorders. 34(3-4):143-148.

Canet J, Raeder J, Rasmussen LS, et al. 2003. Cognitive dysfunction after minor surgery in the elderly. Acta anaesthesiologica Scandinavica. 47(10):1204-1210.

Di Tomasso N, Monaco F, Landoni G. 2015. Hepatic and renal effects of cardiopulmonary bypass. Best practice \& research Clinical anaesthesiology. 29(2):151-161.

Evered L, Scott DA, Silbert B, Maruff P. 2011. Postoperative cognitive dysfunction is independent of type of surgery and anesthetic. Anesth Analg. 112(5):1179-1185.

Folstein MF, Folstein SE, McHugh PR. 1975. "Mini-mental state". A practical method for grading the cognitive state of patients for the clinician. J Psychiatr Res. 12(3):189-198.

Gao L, Taha R, Gauvin D, Othmen LB, Wang Y, Blaise G. 2005. Postoperative cognitive dysfunction after cardiac surgery. Chest. 128(5):3664-3670.
Gottesman RF, Grega MA, Bailey MM, et al. 2010. Delirium after coronary artery bypass graft surgery and late mortality. Annals of neurology. 67(3):338-344.

Hessel EA, 2nd. 2015. History of cardiopulmonary bypass (CPB). Best practice \& research Clinical anaesthesiology. 29(2):99-111.

Heyer EJ, Sharma R, Rampersad A, et al. 2002. A controlled prospective study of neuropsychological dysfunction following carotid endarterectomy. Archives of neurology. 59(2):217-222.

Hudetz JA, Gandhi SD, Iqbal Z, Patterson KM, Pagel PS. 2011. Elevated postoperative inflammatory biomarkers are associated with shortand medium-term cognitive dysfunction after coronary artery surgery. J Anesth. 25(1):1-9.

Iasonos A, Schrag D, Raj GV, Panageas KS. 2008. How to build and interpret a nomogram for cancer prognosis. Journal of clinical oncology: official journal of the American Society of Clinical Oncology. 26(8):1364-1370.

Kalman J, Juhasz A, Bogats G, et al. 2006. Elevated levels of inflammatory biomarkers in the cerebrospinal fluid after coronary artery bypass surgery are predictors of cognitive decline. Neurochemistry international. 48(3):177-180.

Kawai K, Ishihara S, Yamaguchi H, et al. 2015. Nomogram prediction of metachronous colorectal neoplasms in patients with colorectal cancer. Annals of surgery. 261(5):926-932.

Koster S, Hensens AG, Schuurmans MJ, van der Palen J. 2012. Consequences of delirium after cardiac operations. The Annals of thoracic surgery. 93(3): 705-711

Koster S, Oosterveld FG, Hensens AG, Wijma A, van der Palen J. 2008. Delirium after cardiac surgery and predictive validity of a risk checklist. The Annals of thoracic surgery. 86(6):1883-1887.

Launer LJ, Dinkgreve MA, Jonker C, Hooijer C, Lindeboom J. 1993. Are age and education independent correlates of the Mini-Mental State Exam performance of community-dwelling elderly? Journal of gerontology. 48(6):P271-277.

Mangusan RF, Hooper V, Denslow SA, Travis L. 2015. Outcomes associated with postoperative delirium after cardiac surgery. American journal of critical care: an official publication, American Association of CriticalCare Nurses. 24(2):156-163.

Monk TG, Weldon BC, Garvan CW, et al. 2008. Predictors of $\operatorname{cog}$ nitive dysfunction after major noncardiac surgery. Anesthesiology. 108(1):18-30.

Nasreddine ZS, Phillips NA, Bedirian V, et al. 2005. The Montreal Cognitive Assessment, MoCA: a brief screening tool for mild cognitive impairment. J Am Geriatr Soc. 53(4):695-699.

Rasmussen LS. 2006. Postoperative cognitive dysfunction: incidence and prevention. Best practice \& research Clinical anaesthesiology. 20(2):315-330.

Richards M, Meade TW, Peart S, Brennan PJ, Mann AH. 1997. Is there any evidence for a protective effect of antithrombotic medication on cognitive function in men at risk of cardiovascular disease? Some preliminary findings. Journal of neurology, neurosurgery, and psychiatry. 62(3):269-272.

Shaw S, Wang X, Redd H, Alexander GD, Isales CM, Marrero MB. 2003. High glucose augments the angiotensin II-induced activation of JAK2 in vascular smooth muscle cells via the polyol pathway. The Journal of biological chemistry. 278(33):30634-30641.

Silverstein JH, Timberger M, Reich DL, Uysal S. 2007. Central nervous 
system dysfunction after noncardiac surgery and anesthesia in the elderly. Anesthesiology. 106(3):622-628.

van Harten AE, Scheeren TW, Absalom AR. 2012. A review of postoperative cognitive dysfunction and neuroinflammation associated with cardiac surgery and anaesthesia. Anaesthesia. 67(3):280-293.

Wan Y, Xu J, Ma D, Zeng Y, Cibelli M, Maze M. 2007. Postoperative impairment of cognitive function in rats: a possible role for cytokine-mediated inflammation in the hippocampus. Anesthesiology. 106(3):436-443.

Wan Y, Xu J, Meng F, et al. 2010. Cognitive decline following major surgery is associated with gliosis, beta-amyloid accumulation, and tau phosphorylation in old mice. Critical care medicine. 38(11):2190-2198.

Wu Y, Chen T. 2016. An Up-to-Date Review on Cerebral Microbleeds. Journal of stroke and cerebrovascular diseases : the official journal of National Stroke Association. 25(6):1301-1306.

Yuan SM. 2012. Cardiac surgical procedures for the coronary sequelae of Kawasaki disease. The Libyan journal of medicine. 7:19796.

Zhang WY, Wu WL, Gu JJ, et al. 2015. Risk factors for postoperative delirium in patients after coronary artery bypass grafting: A prospective cohort study. Journal of critical care. 30(3):606-612. 